\title{
J. FONLUPT
}

\section{L'affectation exponentielle et le problème du plus court chemin dans un graphe}

Revue française d'automatique, d'informatique et de recherche opérationnelle. Recherche opérationnelle, tome 15, $\mathrm{n}^{\circ} 2$ (1981), p. 165-184.

$<$ http://www.numdam.org/item?id=RO_1981_15_2_165_0>

(C) AFCET, 1981, tous droits réservés.

L'accès aux archives de la revue « Revue française d'automatique, d'informatique et de recherche opérationnelle. Recherche opérationnelle » implique l'accord avec les conditions générales d'utilisation (http://www.numdam.org/ legal.php). Toute utilisation commerciale ou impression systématique est constitutive d'une infraction pénale. Toute copie ou impression de ce fichier doit contenir la présente mention de copyright.

\section{Numdam}

Article numérisé dans le cadre du programme

Numérisation de documents anciens mathématiques

http://www.numdam.org/ 


\title{
L'AFFECTATION EXPONENTIELLE ET LE PROBLĖME DU PLUS COURT CHEMIN DANS UN GRAPHE ( $\left.{ }^{*}\right)$
}

\author{
par J. Fonlupt $\left({ }^{1}\right)$
}

Résumé. - Étant donné un réseau routier et un trafic circulant entre une origine et une destination, nous étudions un modèle d'affectation de trafic introduit par Dial. Nous montrons que cette affectation est la solution d'un programme convexe; cette solution s'obtient d'une manière particulièrement simple, par résolution de systèmes d'équations linéaires.

Ce programme se déduit du programme linéaire décrivant le problème du plus court chemin en ajoutant à la fonction objectif de ce dernier une fonction pénalité du type entropie. Ce programme permet d'étudier sous un angle nouveau les relations entre algorithmes de plus court chemin et de résolution de systèmes linéaires.

Mots clés : Graphes, programme convexe, transport.

Abstract. - Given a road network and a traffic going from an origin to a final destination, we study a model of traffic assignment introduced by Dial. We show that this traffic assignment can be found by solving a convex program; the solution of this program can be obtained very easily by solving systems of linear equations.

This program can be deduced from the linear program describing the shortest path problem by the addition of a penalty function (which is an entropy function) to the objective function. This convex program gives a new insight in the relations between shortest path algorithms and method of resolutions of systems of linear equations.

Keywords: Graphs, convex program, transportation.

\section{INTRODUCTION}

Soit un graphe $G=(V, E) ; V=\left\{v_{1}, v_{2}, \ldots, v_{n}\right\} ; E=\left\{e_{1}, e_{2}, \ldots, e_{m}\right\}$. Les sommets $v_{1}$ et $v_{n}$ seront appelés respectivement sommet origine et sommet destination de $G$. A chaque arc $e_{i} \in E$ est associé un coût $c\left(e_{i}\right)$.

Le graphe $G$ représentera un réseau routier, les sommets $\left\{v_{i}\right\} \in V$ étant les nœuds du réseau et les arcs $\left\{e_{i}\right\} \in E$ étant les tronçons du réseau, reliant certaines paires de nœuds. Le coût $c\left(e_{i}\right)$ de l'arc $e_{i}$ représente, par exemple le temps nécessaire pour relier le nœud origine au nœud extrémité de $e_{i}$.

(*) Reçu septembre 1979.

( $\left.{ }^{1}\right)$ Université scientifique et médicale de Grenoble, B. P. n ${ }^{\circ}$ 53, 38041 Grenoble Cedex.

R.A.I.R.O. Recherche opérationnelle/Operations Research, 0399-0559/1981/165/\$5.00

(C) AFCET-Bordas-Dunod 
Considérons un trafic d'intensité $F$ se rendant du sommet origine $v_{1}$ au sommet destination $v_{n}$. Une affectation sera une répartition du trafic entre tous les itinéraires reliant $v_{1}$ à $v_{n}$. L'affectation suivant le plus court chemin noté $A_{0}$ sera celle où tout le trafic emprunte un plus court chemin entre $v_{1}$ et $v_{n}$. Dans [4] Dial a étudié un autre type d'affectation dans le cas où $G$ est sans circuit. Nous appellerons cette affectation, affectation exponentielle suivant en cela une terminologie utilisée dans [6]. Cette affectation notée $A_{\lambda}$ dépend d'un paramètre $\lambda$ et quand $\lambda \rightarrow 0, A_{\lambda}$ a pour limite l'affectation $A_{0}$.

Le but essentiel de cette publication est d'étudier dans le cas général l'affectation exponentielle et de montrer que celle-ci peut se trouver comme solution d'un programme d'optimisation convexe qui se résoud d'une manière particulièrement simple.

Au paragraphe II nous reviendrons sur les méthodes de Dial quand le graphe $G$ est sans circuit.

Au paragraphe III, nous étudierons l'affectation exponentielle dans le cas général.

Au paragraphe IV, nous étudierons, sous un angle nouveau, les relations entre résolutions de systèmes d'équations linéaires et algorithmes de plus courts chemins.

\section{L'ALGORITHME DE DIAL}

\section{Défınitions générales}

Un chemin $t$ sera défini par la succession de ses arcs : $t=\left[e_{i_{1}}, \ldots, e_{i_{k}}\right] . T(1, n)$ sera l'ensemble des chemins joignant l'origine $v_{1}$ à la destination $v_{n} . T\left(1, n ; e_{i}\right)$ sera l'ensemble des chemins d'origine $v_{1}$, d'extrémité $v_{n}$ et empruntant l'arc $e_{i}$. La longueur $c(t)$ d'un chemin sera définie par :

$$
c(t)=\sum_{e_{i} \in t} c\left(e_{i}\right)
$$

2. Nous ferons dans ce paragraphe les hypothèses suivantes :

(a) le graphe $G$ est sans circuit;

(b) $\forall$ le sommet $v_{i} \in V, \exists$ un chemin $t \in T(1, n)$ passant par ce sommet.

Il est facile de montrer que les conditions $(a)$ et $(b)$ sont équivalentes aux conditions suivantes :

$\left(a^{\prime}\right) \exists$ un ordre partiel noté $\leqq$ sur les sommets du graphe défini comme il suit : $v_{i} \leqq v_{j} \Leftrightarrow \exists$ un chemin d'origine $v_{i}$ et d'extrémité $v_{j}$ (on ne peut avoir $v_{i}<v_{j}$ et $\left.v_{j}<v_{i}\right)$;

(b') $v_{1} \leqq v_{i} \leqq v_{n}, \forall v_{i} \in V$. 
On peut alors supposer en reclassant éventuellement les sommets du graphe que : $v_{i}<v_{j} \Rightarrow i<j$.

3. L'affectation exponentielle $A_{\lambda}$ de paramètre $\lambda$ peut se définir de la manière suivante :

Chargeons chaque itinéraire $t \in T(1, n)$ d'un trafic $r(t)$ égal à :

$$
r(t)=\mu e^{-c(t) / \lambda}
$$

La constante sera définie de telle façon que la somme de tous les trafics sur les chemins $t \in T(1, n)$ est égale à l'intensité $F$ du trafic total entrant par $v_{1}$ :

$$
\mu \sum_{t \in T(1, n)} r(t)=F
$$

Les formules (1) et (2) définissent l'affectation exponentielle $A_{\lambda}$ de paramètre $\lambda$. $A_{\lambda}$ définit sur chaque arc $e \in E$ un trafic noté $x_{\lambda}(e)$ qui se calcule par la formule :

$$
x_{\lambda}(e)=\sum_{t \in T[1, n ; e]} \mu r(t) .
$$

Notons que les formules (3) ne sont pas intéressantes d'un point de vue algorithmique car elles nécessitent pour chaque arc $e \in E$ une énumération de tous les chemins $t \in T[1, n ; e]$.

L'algorithme de Dial permet cependant de calculer d'une façon efficace les quantités $x_{\lambda}(e)$.

\section{Algorithme de Dial}

Notations : si $e$ est un arc d'extrémité initial $v_{i}$ et d'extrémité finale $v_{j}$, on. posera :

$$
i=\operatorname{or}(e) ; \quad j=\operatorname{ex}(e) .
$$

D'autre part, posons $\overline{c_{\lambda}}(e)=e^{-c(e) / \lambda}, \forall e \in E$ :

$$
\begin{array}{ll}
\Omega^{-}(i)=\{e \in E ; \operatorname{ex}(e)=i\}, & \forall i=1, \ldots, n ; \\
\Omega^{+}(i)=\{e \in E ; \text { or }(e)=i\}, & \forall i=1, \ldots, n .
\end{array}
$$

Enfin à chaque sommet $v_{i}$, on associera deux fonctions $v_{i} \rightarrow \omega(i)$ et $v_{i} \rightarrow \omega^{\prime}(i)$ définies de la manière suivante : 
Calcul de $\omega$

$$
\left.\begin{array}{c}
\omega(1)=1, \\
\omega(i)=\sum_{e \in \Omega^{-}(i)} \bar{c}_{\lambda}(e) \omega[\text { or }(e)], \quad i=2, \ldots, n .
\end{array}\right\}
$$

Notons que si $e \in \Omega^{-}(i)$, or $(e)<i$ à cause de la numérotation des sommets adoptés au paragraphe 2 .

La fonction $\omega$ se calcule donc en balayant les sommets du graphe dans un ordre croissant.

Calcul de $\omega^{\prime}$

De même la fonction $\omega^{\prime}$ se calcule en balayant les sommets de $G$ dans un ordre décroissant par les formules :

$$
\left.\begin{array}{c}
\omega^{\prime}(n)=1, \\
\omega^{\prime}(i)=\sum_{e \in \Omega^{+}(i)} \bar{c}_{\lambda}(e) \omega^{\prime}[e x(e)], \quad 1 \leqq i \leqq n-1 .
\end{array}\right\}
$$

THÉORÈME 1 (Dial) :

$$
x_{\lambda}(e)=\frac{F \cdot \bar{c}_{\lambda}(e) \omega[o r(e)] \cdot \omega^{\prime}[e x(e)]}{\omega(n)} .
$$

Démonstration : Voir (4) ou (7) pour une démonstration plus mathématique.

\section{Réciproque du théorème de Dial}

Soit $X_{\lambda}=\left[x_{\lambda}(e) \mid e \in E\right]$ un vecteur. $X_{\lambda}$ sera appelé vecteur trafic d'intensité $F$ ou trafic d'intensité $F$ si :

$$
\left\{\begin{aligned}
& X_{\lambda} \geqq 0, \\
& y(1)=\sum_{e \in \Omega^{+}(1)} x_{\lambda}(e)=F, \\
& y(i)=\sum_{e \in \Omega^{+}(i)} x_{\lambda}(e)=\sum_{e \in \Omega^{-}(i)} x_{\lambda}(e), \quad i=2, \ldots, n-1, \\
& y(n)=\sum_{e \in \Omega^{-}(n)} x_{\lambda}(e)=F,
\end{aligned}\right.
$$

[y(i) représente le trafic passant par le nnud $i]$.

Le théorème de Dial fournit une représentation simple du trafic par arc à partir du trafic défini sur les itinéraires $t \in T(1, n)$.

Le théorème suivant répond à la question inverse :à partir d'un trafic observé $X_{\lambda}$ d'intensité $F$, peut-on trouver un système de coûts $\{c(e): e \in E\}$ tels que l'affectation exponentielle $A_{\lambda}$ fournisse le trafic $X_{2}$ ? 


\section{Posons :}

$$
\begin{array}{ccccc}
\bar{c}_{\lambda}(e)=\frac{x_{\lambda}(e)}{y[e x(e)]} & \text { si } & x_{\lambda}(e)>0 & \text { et } & c(e)=-\lambda \log \bar{c}_{\lambda}(e), \\
\bar{c}_{\lambda}(e)=0 & \text { si } & x_{\lambda}(e)=0 & \text { et } & c(e)=+\infty
\end{array}
$$

Soit $A_{\lambda}$ l'affectation exponentielle de paramètre $\lambda$ sur $G$ avec les coûts définis par (7).

THÉORÈme 2 : L'affectation exponentielle $A_{\lambda}$ produit un trafic égal à $X_{\lambda}$.

Démonstration : Le système d'équations linéaires :

$$
\left\{\begin{array}{c}
\omega(1)=1, \\
\omega(i)=\sum_{e \in \Omega^{-}(i)} \bar{c}_{\lambda}(e) \omega[\operatorname{or}(e)]=\sum_{e \in \Omega^{-}(i)} \frac{x_{\lambda}(e) \omega[\operatorname{or}(e)]}{y[\operatorname{ex}(e)]}=\sum_{e \Omega^{-}(i)} \frac{x_{\lambda}(e) \omega[\operatorname{or}(e)]}{y(i)}
\end{array}\right.
$$

a pour unique solution $\omega(i)=1,1<i \leqq n$.

De même le système :

$$
\left\{\begin{array}{c}
\omega^{\prime}(n)=1, \\
\omega^{\prime}(i)=\sum_{e \in \Omega^{+}(i)} \bar{c}_{\lambda}(e) \omega^{\prime}[\operatorname{ex}(e)]=\sum_{e \in \Omega^{+}(i)} \frac{x_{\lambda}(e) \omega^{\prime}[\operatorname{ex}(e)]}{y[\operatorname{ex}(e)]}
\end{array}\right.
$$

a pour solution unique $\omega^{\prime}[i]=y(i) / F$.

L'affectation exponentielle $A_{\lambda}$ associée aux coûts $c(e)$ définis en (7) produit sur chaque arc un trafic :

$$
x_{\lambda}^{\prime}(e)=F \bar{c}_{\lambda}(e) \frac{\omega[\operatorname{or}(e)] \omega^{\prime}[\operatorname{ex}(e)]}{\omega(n)}=x_{\lambda}(e)
$$

C.Q.F.D.

\section{Remarque}

Soit $I$ la matrice identité de dimension $n \times n$ et soit $A=\left\|a_{i j}\right\|$ la matrice $n \times n$ définie par:

$$
a_{i i}=0 .
$$

Si aucun arc ne joint le sommet $v_{i}$ au sommet $v_{j} a_{i j}=0$.

Si $\exists$ un arc $e$ tel que or $(e)=i$ et $\operatorname{ex}(e)=j, a_{i j}(\lambda)=\bar{c}_{\lambda}(e)$. Notons que $A(\lambda)$ est une matrice triangulaire supérieure d'après la numérotation des sommets (voir § 2). 
Posons :

$$
\begin{gathered}
u_{1}^{T}=[1,0, \ldots, 0,0], \\
u_{n}^{T}=[0,0, \ldots, 0,1], \\
\omega=[\omega(i) ; i=1, \ldots, n], \\
\omega^{\prime}=\left[\omega^{\prime}(i) ; i=1, \ldots, n\right] .
\end{gathered}
$$

Le système d'équations (4) peut s'écrire :

$$
\left[I-A^{T}(\lambda)\right] \omega=u_{1} .
$$

Le système d'équations (5) peut s'écrire :

$$
[I-A(\lambda)] \omega^{\prime}=u_{n} \text {. }
$$

\section{L'AFFECTATION EXPONENTIELLE DANS LE CAS GÉNÉRAL}

\section{Notations et rappels}

Dans l'espace $R^{n}$, posons :

$$
R_{+}^{n}=\left\{x \in E \mid x_{i} \geqq 0, \forall i \in[1, n]\right\} \quad \text { et } \quad R_{++}^{n}=\left\{x \in E \mid x_{i}>0, \forall i \in[1, n]\right\} .
$$

Considérons la fonction :

$$
g_{0}(x)=\sum_{i=1}^{n} x_{i} \log \frac{x_{i}}{\sum_{i=1}^{n} x_{i}} .
$$

La fonction $g_{0}(x)$ est définie et continue pour $x \in R_{++}^{n}$ mais en posant 0 $\log 0=0$ et $g_{0}(0)=0$ on définit $g_{0}(x)$ pour $x \in R_{+}^{n}$.

La fonction $g_{0}(x)$ a déjà été étudiée dans un autre contexte. C'est par exemple la fonction entropie d'un système dans le problème de l'équilibre chimique (voir [2], p. 479) (voir également [6]).

Rappelons que la fonction $h(x)=\sum_{i=1}^{n} x_{i} \log x_{i}$ est définie, continue pour $x \in R_{+}^{n}$. D'autre par $h(x)$ est strictement convexe et dérivable pour $x \in R_{++}^{n}$. Son vecteur dérivé en $x: \partial h / \partial x(x)$ défini par ses composantes :

$$
u_{i}(x)=\frac{\partial h(x)}{\partial x_{i}}=\left(\log x_{i}\right)+1
$$

est l'unique sous-gradient de $h(x)$ en $x$. (Pour la définition et les propriétés des sous-gradients voir Rockafellar [9].) Comme $u_{i}(x) \rightarrow-\infty$ quand $x_{i} \rightarrow 0, h(x)$ 
n'admet pas de sous-gradient pour $x \in R_{+}^{n} ; x \notin R_{++}^{n}$. Ces résultats élémentaires s'étendent à la fonction $g_{0}$ de la manière suivante :

PRoposition $1:(a) g_{0}(x)$ est définie et continue pour $x \in R_{+}^{n}$.

(b) $g_{0}(x)$ est homogène et convexe pour $x \in R_{++}^{n}$.

Si $x, y, z \in R_{+}^{n}$ sont tels que $z=\alpha x+\beta y$ avec $(\alpha, \beta \geqq 0, \alpha+\beta=1)$ et $g(z)=\alpha g(x)+\beta g(y) x, y, z$ sont proportionnels.

(c) $g_{0}(x)$ est dérivable pour $x \in R_{++}^{n}$ et son vecteur dérivée en $x: \partial g_{0}(x) / \partial x$ défini par ses composantes $\partial g_{0}(x) / \partial x_{i}=\log x_{i} / \sum_{i=1}^{n} x_{i}$ est l'unique sous-gradient de $g_{0}$ en $x$.

$g_{0}$ n'admet pas de sous-gradient pour $x \in R_{++}^{n}, x \notin R_{+}^{n} ; x \neq 0$. Pour $x=0$, l'ensemble des sous-gradients de $g_{0}: \partial f(0)$ est égal à

$$
\partial f(0)=\left[\operatorname{conv}\left\{\partial g_{0}(x) / \partial x\right\} \mid x \in R_{++}^{n}\right] .
$$

Considérons la fonction :

$$
g_{1}(x)=g_{0}(x)+[(\log n)+1]\left[\sum_{i=1}^{n} x_{i}\right]
$$

$g_{1}$ étant somme de $g_{0}$ et d'une fonction linéaire, les propriétés résumées dans la proposition 1 s'étendent à $g_{1}$ avec en plus les résultats suivants :

Proposition 1' : $(a) g_{1}(x) \geqq \sum_{i=1}^{n} x_{i} \geqq 0$ pour $x R_{+}^{n}, g_{1}(0)=0$.

(b) Si $g_{1}(x)=g_{1}(y)$ pour $x \neq y$, $x$ et $y$ ne peuvent être proportionnels et :

$$
\begin{gathered}
\forall z=\alpha x+\beta y ; \quad \alpha \geqq 0, \quad \beta \geqq 0, \quad \alpha+\beta=1, \\
g_{1}(z)<g_{1}(x)=g_{1}(y) .
\end{gathered}
$$

Démonstration : (a) Soit :

$$
\bar{X}=\left[x_{i}=1, \forall 1 \leqq i \leqq n\right], \quad \frac{\partial g_{0}(\bar{X})}{\partial x}=\left[\frac{\partial g_{0}(\bar{X})}{\partial x_{i}}=-\log n\right]
$$

Par la propriété $1(c)$ :

$$
\frac{\partial g_{0}(\bar{X})}{\partial x} \in \partial f(0)
$$

vol. $15, \mathrm{n}^{\circ} 2$, mai 1981 
Par les propriétés des sous-gradients :

$$
\begin{aligned}
g_{0}(x)-g_{0}(0) & \geqq\left\langle\frac{\partial g_{0}(\bar{X})}{\partial x}, x\right\rangle, \quad \forall x \in R_{+}^{n} \\
& \Rightarrow g_{0}(x)+\left(\sum_{i=1}^{n} x_{i}\right) \log n \geqq 0 \Rightarrow g_{1}(x) \geqq \sum_{i=1}^{n} x_{i}, \quad \forall x \in R_{+}^{n} .
\end{aligned}
$$

(b) La fonction $g_{1}$ étant homogène, si $g_{1}(x)=g_{1}(y) x$ et $y$ ne peuvent être proportionnels à moins que $x=y=0$. La propriété $(b)$ de la proposition 1 conduit au résultat.

\section{L'affectation exponentielle}

(a) Tout secteur trafic entre $v_{1}$ et $v_{n} \mathrm{X}=[x(e) ; e \in E]$ satisfait aux contraintes :

$$
\left\{\begin{aligned}
X & \geqq 0, \\
y(1)=\sum_{e \in \Omega^{+}(1)} x(e) & =\sum_{e \in \Omega^{-}(1)} x(e)+F, \\
y(i) & =\sum_{e \in \Omega^{-}(i)} x(e)=\sum_{e \in \Omega^{+}(i)} x(e), \\
y(n)=\sum_{e \in \Omega^{-}(n)} x(e) & =\sum_{e \in \Omega^{+}(n)} x(e)+F .
\end{aligned}\right.
$$

Soit $(P)$ le polyèdre décrit par ces contraintes. Il est bien connu que les points extrêmes de $(P)$ sont les vecteurs représentatifs des chemins élémentaires joignant $v_{1}$ à $v_{n}$. Un plus court chemin entre $v_{1}$ et $v_{n}$ est donc une solution du programme linéaire :

$$
L_{0}\left\{\begin{array}{c}
X \in P, \\
z_{0}=\min z_{0}(x)=\min \sum_{e \in E} c(e) x(e),
\end{array}\right.
$$

$(P)$ n'étant pas borné, la condition nécessaire et suffisante pour que $L_{0}$ ait une solution finie est qu'il n'y ait pas dans $G$ de circuit absorbant, c'est-à-dire de circuit de longueur négative. Cette hypothèse que nous appellerons condition (A) sera supposée réalisée dans toute la suite de cet article. Soit $X_{0}$ la solution de $L_{0}$. Quand $X_{0}$ n'est pas unique, on notera $\left[X_{0}\right]$ l'ensemble des solutions de $L_{0}$. Nous ferons pour la démonstration des théorèmes (4) et (6) l'hypothèse suivante : Condition (B) : $\forall v_{i} \in V, \exists$ un chemin joignant $v_{1}$ à $v_{n}$ et passant par $v_{i}$. Il est facile de montrer que la condition (B) implique (B') : $\exists X \in P$ vérifiant $X>0$.

Pour chaque sommet $v_{i} 2 \leqq i \leqq n$, posons :

$$
f_{i}(x)=\sum_{e \in \Omega^{-}(i)} x(e) \log \frac{x(e)}{y(i)}+[1+\log n] y(i) .
$$

R.A.I.R.O. Recherche opérationnelle/Operations Research 
Pour $i=1$,

$$
f_{1}(X)=F \log \frac{F}{y(1)}+\sum_{e \in \Omega^{-}(1)} x(e) \log \frac{x(e)}{y(1)}+(1+\log n) y(1) .
$$

Enfin posons $f_{\lambda}(X)=\lambda \sum_{i=1}^{n} f_{i}(X)$.

Les fonctions $X \rightarrow f_{i}(X)(i=1, \ldots, n)$ satisfont les propositions (1) et $\left(1^{\prime}\right)$. Il en est donc de même de $f_{\lambda}(X)$.

Soit $L_{\lambda}$ le programme convexe :

$$
\left\{\begin{array}{c}
X \in P \\
z_{\lambda}=\min z_{\lambda}(X)=\min \left[f_{\lambda}(X)+\sum_{e \in E} c(e) x(e)\right]
\end{array}\right.
$$

Pour $\lambda=0$ on retrouve le programme $L_{0}$.

THÉORÈME $4:$ (a) $\forall \lambda>0$ le programme $L_{\lambda}$ a une solution unique $X_{\lambda}$.

(b) La fonction $\lambda \rightarrow\left(X_{\lambda}, z_{\lambda}\right)$ est continue pour $\lambda>0$.

La fonction $\lambda \rightarrow z_{\lambda}$ est décroissante.

Quand $\lambda \rightarrow 0, z_{\lambda} \rightarrow z_{0}$ et $X_{\lambda} \rightarrow X_{0} \in\left[X_{0}\right]$.

(c) $X_{\lambda}>0$.

Démonstration : (a) Par la proposition 1':

$$
f_{i}(X) \geqq y(i), \quad \forall X \geqq 0, \quad \forall i \in[1, n] .
$$

Donc :

$f_{\lambda}(X) \geqq \lambda\left[\sum_{i=1}^{n} y(i)\right] \Rightarrow \forall X \in P, \quad z_{\lambda}(X) \geqq z_{0}(X) \Rightarrow z_{\lambda} \geqq z_{0}$ et $z_{\lambda}$ existe.

Soit. $X_{0}=\left[x_{0}(e): e \in E\right]$ un vecteur représentatif d'un plus court chemin. élémentaire $t_{0}$ entre $v_{1}$ et $v_{n}:\left[x_{0}(e)=F\right.$ si $e \in t_{0}$ et $x_{0}(e)=0$ si $\left.e \notin t_{0}\right]$.

On vérifie alors que

$$
\forall i \in[1, n], \quad \sum_{e \in \Omega^{-}(i)} x_{0}(e) \log \frac{x_{0}(e)}{y_{0}(i)}=0 .
$$

$$
\begin{aligned}
\text { En posant } y_{0}(i)= & \sum_{e \in \Omega^{-}(i)} x_{0}(e), \\
& z_{\lambda}\left(X_{0}\right)=\lambda(1+\log n)\left[\sum_{i=1}^{n} y_{0}(i)\right]+z_{0} .
\end{aligned}
$$


Comme d'autre part :

pour $X \in P$ vérifiant :

$$
z_{\lambda}(X) \geqq \lambda\left[\sum_{i=1}^{n} y(i)\right]+z_{0}, \quad \forall X \in P,
$$

$$
\sum_{i=1}^{n} y(i)>\lambda\left[\sum_{i=1}^{n} y_{0}(i)\right](1+\log n)
$$

on aura $z_{\lambda}(X)>z_{\lambda}\left(X_{0}\right)$.

Posons :

$$
P^{\prime}=P \cap\left[X: \sum_{i=1}^{n} y(i) \leqq \lambda\left[\sum_{i=1}^{n} y_{0}(i)\right](1+\log n)\right]
$$

$P^{\prime}$ est un polyèdre borné donc compact et on a :

$$
\operatorname{Inf}\left[z_{\lambda}(X) \mid X \in P\right]=\operatorname{Inf}\left[z_{\lambda}(X) \mid X \in P^{\prime}\right]=\min \left[z_{\lambda}(X) \mid X \in P^{\prime}\right] .
$$

Ce qui prouve que $X_{\lambda}$ existe et $X_{\lambda} \in P^{\prime}$. L'unicité de $X_{\lambda}$ se déduit aisément de la proposition $1^{\prime}(b)$.

(b) Comme $X_{\lambda} \in P^{\prime}, \forall \lambda \geqq 0$, les résultats de (b) sont des applications de théorèmes classiques d'analyse sur le comportement du minimum d'une fonction continue dépendant d'un paramètre $\lambda$ sur un ensemble compact.

(c) C'est le point important du théorème.

Supposons (c) faux. Soit $e_{0} \in E$ tel que $x_{\lambda}\left(e_{0}\right)=0$.

Soit un chemin $t=\left[e_{0}, e_{1}, \ldots, e_{k}\right]$ dont le premier arc est $e_{0}$ et le dernier arc $e_{k}$ a pour extrémité finale $v_{n}$. Parcourant $t$ en partant de $e_{0}$ vers $e_{k}$, on trouvera un arc noté $e$ d'extrémité $v_{i}$ tel que $x_{\lambda}(e)=0$ et $y(i)>0$ [puisque $x_{\lambda}\left(e_{0}\right)=0$ et $\left.y(n)=\sum_{e \in \Omega^{-}(n)} x(e)>0\right]$

Mais comme par $\left(B^{\prime}\right) \exists$ une solution $\bar{X}>0$, par les théorèmes d'optimalité des programmes convexes (voir [9], p. 281, théorème 28.2) $X_{\lambda}$ ne peut être solution. de $L_{\lambda}$ puisque $z_{\lambda}(X)$ n'admet pas de sous-gradient en un point $X_{\lambda}$ tel que $\left[x_{\lambda}(e)=0\right.$ et $\left.y(i)>0\right]$.

$\Rightarrow$ Donc $X_{\lambda}>0$.

DéfINITION : L'affectation exponentielle $A_{\lambda}$ de paramètre $\lambda>$ entre $v_{1}$ et $v_{n}$ est le vecteur trafic $X_{\lambda}$ solution du programme $L_{\lambda}$.

Posons pour chaque sommet $v_{i}(i \leqq i<n)$ :

$$
f_{i}^{\prime}(X)=\sum_{e \in \Omega^{+}(i)} x(e) \log \frac{x(e)}{y(i)}+(1+\log n) y(i) .
$$

R.A.I.R.O. Recherche opérationnelle/Operations Research 
Pour $i=n$ :

$$
\begin{gathered}
f_{n}^{\prime}(X)=F \log \frac{F}{y(n)}+\sum_{e \in \Omega^{+}(n)} x(e) \log \frac{x(e)}{y(n)}+(1+\log n) y(n) \\
f_{\lambda}^{\prime}(X)=\lambda \sum_{i=1}^{n} f_{i}^{\prime}(X) . \\
z_{\lambda}^{\prime}(X)=f_{\lambda}^{\prime}(X)+\sum_{e \in E} c(e) x(e) .
\end{gathered}
$$

Lemme 5 :

$$
\forall X \in P, \quad z_{\lambda}(X)=z_{\lambda}^{\prime}(X) .
$$

Démonstration : Soit $X \in P$ :

$$
\begin{aligned}
& f_{\lambda}(X)=\lambda\left[F \log \frac{F}{y(1)}+\sum_{e \in E} x(e) \log x(e)\right. \\
& \left.+\sum_{i=1}^{n} \sum_{e \in \Omega^{-}(i)} x(e) \log y(i)\right]+\lambda[1+\log n]\left[\sum_{i=1}^{n} y(i)\right], \\
& f_{\lambda}(X)=\lambda\left[F \log F+\sum_{e \in E} x(e) \log x(e)\right. \\
& \left.+\sum_{i=1}^{n} y(i) \log y(i)\right]+\lambda[(1+\log n)]\left[\sum_{i=1}^{n} y(i)\right] \\
& f_{\lambda}(X)=\lambda\left[F \log \frac{F}{y(n)}+\sum_{e \in E} x(e) \log x(e)\right. \\
& \left.+\sum_{i=1}^{n} \sum_{e \in \Omega^{+}(i)} x(e) \log y(i)\right]+\lambda(1+\log n)\left[\sum_{i=1}^{n} y(i)\right]=f_{\lambda}^{\prime}(X) . \\
& \text { C.Q.F.D. }
\end{aligned}
$$

\section{Résolution de $L_{\lambda}$}

Nous poserons :

$$
\bar{c}_{\lambda}(e)=e^{-(c(e) / \lambda)-(1+\log n)}
$$

La matrice $A(\lambda) \in R^{n \times n}$ sera définie par : $a_{i i}=0$.

Si il n'existe aucun arc joignant $v_{i}$ à $v_{j} a_{i j}=0$.

Si il existe $e$ tel que or $(e)=v_{i}$ et $\operatorname{ex}(e)=v_{j} a_{i j}=\bar{c}_{\lambda}(e)$.

Posons :

$$
\begin{gathered}
u_{1}^{T}=[1,0, \ldots, 0,0], \quad u_{n}^{T}=[0,0, \ldots, 0,1], \\
\omega=[\omega(i) ; i=1, \ldots, n], \quad \omega^{\prime}=\left[\omega^{\prime}(i) ; i=1, \ldots, n\right] .
\end{gathered}
$$


THÉORÈME $6:(a)$ Le système linéaire $\left[I-A^{T}(\lambda)\right] \omega=u_{1}$ a une solution unique $\omega>0$.

Le système linéaire $[I-A(\lambda)] \omega^{\prime}=u_{n}$ a une solution unique $\omega^{\prime}>0$.

La solution $X_{\lambda}$ de $L_{\lambda}$ est donnée par :

$$
x_{\lambda}(e)=F \frac{\bar{c}_{\lambda}(e) \omega[\operatorname{or}(e)] \omega^{\prime}[\operatorname{ex}(e)]}{\omega(n)} .
$$

(b) La valeur de $L_{\lambda}$ est $z_{\lambda}=-\lambda F \log \omega(n)+\lambda F(1+\log n)$.

Démonstration: (a) Par le théorème $4, X_{\lambda}$ existe et $X_{\lambda}>0$. Si $\left\{\partial z_{\lambda}\left(X_{\lambda}\right) / \partial x(e)\right.$; $e \in E\}$ est le gradient de $z_{\lambda}(X)$ en $X_{\lambda}$, par les théorèmes d'optimalité des programmes convexes ([9], p. 283, théorème de Kuhn et Tucker), à chaque contrainte de $(P)$ décrivant la conservation du trafic en $\left\{u_{i}\right\}$, on peut associer une variable duale optimale notée $\mu(i)$ et les conditions de Kuhn et Tucker s'écrivent :

$$
x_{\lambda}(e)\left[\frac{\partial z_{\lambda}\left(X_{\lambda}\right)}{\partial x(e)}-\mu[o r(e)]+\mu[e x(e)]\right]=0, \quad \forall e \in E .
$$

Comme $x_{\lambda}(e)>0$ par le théorème $4(c), \forall e \in E$, on a :

$$
\frac{\partial z_{\lambda}\left(X_{\lambda}\right)}{\partial x(e)}=\log \frac{x_{\lambda}(e)}{y_{\lambda}[\operatorname{ex}(e)]}+1+\log n+\frac{1}{\lambda} c(e)=+\mu[\operatorname{or}(e)]-\mu[\operatorname{ex}(e)]
$$

en notant $y_{\lambda}(i), 1 \leqq i \leqq n$ le trafic passant par le nœud $i$ dans la solution optimale $X_{\lambda}$.

Posons $\omega(i)=e^{\mu(i)}, i \in[1, n]$.

Notons que $\omega=[\omega(i) ; i \in[1, n]]>0$.

Les relations (1) s'écrivent :

$$
\forall e \in E, \frac{x_{\lambda}(e)}{y_{\lambda}[\operatorname{ex}(e)]}=\bar{c}_{\lambda}(e) \frac{\omega[\operatorname{or}(e)]}{\omega[\operatorname{ex}(e)]} .
$$

Posons d'autre part :

$$
\frac{F}{y_{\lambda}(1)}=\frac{\alpha}{\omega(1)} \text {. }
$$

Les relations (2), restant inchangées si on multiplie les variables $\omega(i) ; i \in[1, n]$ par une même quantité, on peut fixer $\alpha$ arbitrairement. Comme le premier membre de (2') est positif et $\omega(1)>0$ on doit prendre $\alpha>0$. Fixons donc $\alpha=1$. 
Considérons parmi les relations (2) et ( $\left.2^{\prime}\right)$ celles associées aux arcs $e \in E$ ayant même extrémité finale $v_{i}(1 \leqq i \leqq n)$ et faisons la somme membre à membre de ces relations.

On trouve alors les relations pour chaque sommet $\left\{v_{i}\right\}$ :

$$
\begin{gathered}
\omega(i)=\sum_{e \in \Omega^{-}(i)} \bar{c}_{\lambda}(e) \omega[\operatorname{or}(e)], \quad i \in[2, n], \\
\omega(1)=\sum_{e \in \Omega^{-}(1)} \bar{c}_{\lambda}(e) \omega[\operatorname{or}(e)]+1 .
\end{gathered}
$$

Par le lemme 5 on peut remplacer dans la fonction objectif $z_{\lambda}(X)$ par $z_{\lambda}^{\prime}(X)$.

Reprenant alors le raisonnement précédent avec la fonction objectif $z_{\lambda}^{\prime}(X)$, il existe des variables duales optimales $\mu^{\prime}(i) i \in[1, n]$ et en posant $\omega^{\prime}(i)=e^{\mu^{\prime}(i)} i \in[1$, $n]$, le vecteur $\omega^{\prime}$ est solution du système.

$$
\begin{gathered}
\omega^{\prime}(i)=\sum_{e \in \Omega^{+}(i)} \bar{c}_{\lambda}(e) \omega^{\prime}[\operatorname{ex}(e)], \quad i \in[1, n-1], \\
\omega^{\prime}(n)=\sum_{e \in \Omega^{+}(n)} \bar{c}_{\lambda}(e) \omega^{\prime}[\operatorname{ex}(e)]+1 .
\end{gathered}
$$

On a donc :

$$
\begin{aligned}
& {\left[I-A^{T}(\lambda)\right] \omega=u_{1},} \\
& {[I-A(\lambda)] \omega^{\prime}=u_{n} .}
\end{aligned}
$$

Considérons le vecteur $X_{\lambda}$ défini par :

$$
x_{\lambda}(e)=F \bar{c}_{\lambda}(e) \frac{\omega[\operatorname{or}(e)] \omega^{\prime}[\operatorname{ex}(e)]}{\omega(n)}
$$

Notons d'abord que $\omega(n)=\omega^{\prime}(1)$ puisque :

$$
\begin{aligned}
\omega^{\prime}(1)=\left\langle\omega^{\prime T}, u_{1}\right\rangle=\left\langle\omega^{\prime T},\left[I-A^{T}(\lambda)\right] \omega\right\rangle & \\
& =\left\langle\omega^{T},(I-A(\lambda)) \omega^{\prime}\right\rangle=\left\langle\omega^{T}, u_{n}\right\rangle=\omega(n) .
\end{aligned}
$$

Pour $i \neq 1$ :

Pour $i=1$ :

$$
\sum_{e \in \Omega^{-}(i)} x_{\lambda}(e)=F \frac{\omega^{\prime}(i)}{\omega(n)} \sum_{e \in \Omega^{-}(i)} \bar{c}_{\lambda}(e) \omega[\operatorname{or}(e)]=\dot{F} \frac{\omega(i) \omega^{\prime}(i)}{\omega(n)} .
$$

Pour $i \neq n$ :

$$
\sum_{e \in \Omega^{-}(1)} x_{\lambda}(e)=F \frac{\omega^{\prime}(1)}{\omega(n)} \sum_{e \in \Omega^{-}(1)} \cdot \bar{c}_{\lambda}(e) \omega[\operatorname{or}(e)]=F[\omega(1)-1]
$$

$$
\sum_{e \in \Omega^{+}(i)} x_{\lambda}(e)=F \frac{\omega(i)}{\omega(n)} \sum_{e \in \Omega^{+}(i)} \bar{c}_{\lambda}(e) \omega^{\prime}[\operatorname{ex}(e)]=F \frac{\omega(i) \omega^{\prime}(i)}{\omega(n)} .
$$

Pour $i=n$ :

$$
\sum_{e \in \Omega^{+}(n)} x_{\lambda}(e)=F \frac{\omega(n)}{\omega(n)} \sum_{e \in \Omega^{+}(n)} \bar{c}_{\lambda}(e) \omega^{\prime}[\operatorname{ex}(e)]=F\left[\omega^{\prime}(n)-1\right] .
$$


Les relations (4) montrent que $X_{\lambda} \in P$. D'autre part, elles montrent que $X_{\lambda}$ satisfait les relations $(2)$ et $\left(2^{\prime}\right)$ c'est-à-dire les relations d'optimalité de Kuhn et Tucker.

Montrons enfin que $\omega$ (resp. $\left.\omega^{\prime}\right)$ est solution unique de (3) [resp. de (3')].

Supposons que (3) n'ait pas une solution unique. Ceci signifie que $\exists$ une solution $\bar{\omega}$ celle que $\left[I-A^{T}(\lambda)\right] \bar{\omega}=0$.

Posons $\omega_{1}=\omega+\varepsilon \bar{\omega}$ avec $\varepsilon$ suffisamment petit pour que $\omega_{1}>0$. $\omega_{1}$ est alors solution de (3).

En posant :

$$
x_{\lambda}^{\prime}(e)=\frac{\omega_{1}[\operatorname{or}(e)] \omega^{\prime}[\operatorname{ex}(e)]}{\omega_{1}[n]}
$$

on vérifie que $X_{\lambda}^{\prime}=\left[x_{\lambda}^{\prime}(e) ; e \in E\right]$ est solution de $L_{\lambda}$, puisque les relations (4) et (2), $\left(2^{\prime}\right)$, sont vérifiées pour $X_{\lambda}^{\prime}, \omega_{1}$ et $\omega^{\prime}$. Mais comme la solution de $L_{\lambda}$ est unique par le théorème (4) $x_{\lambda}^{\prime}(e)=x_{\lambda}(e), \forall e \in E$.

Donc :

$$
\frac{\omega_{1}(i)}{\omega_{1}(n)}=\frac{\omega(i)}{\omega(n)}, \quad \forall i \in[1, n]
$$

Ceci montre que $\omega=\omega^{\prime}$ et on aboutit à une contradiction :

(b) $z_{\lambda}\left(X_{\lambda}\right)=\sum_{e \in E} x_{\lambda}(e)\left[\lambda\left[\log \frac{x_{\lambda}(e)}{y_{\lambda}[\operatorname{ex}(e)]}+1+\log n\right]+c(e)\right]$

$$
+\lambda F\left[\log \frac{F}{y_{\lambda}(1)}+1+\log n\right] \text {. }
$$

Par les relations (1), on 1 :

$$
\begin{gathered}
z_{\lambda}\left(X_{\lambda}\right)=\sum_{e \in E} \lambda x_{\lambda}(e)[\mu \operatorname{or}(e)-\mu[\operatorname{ex}(e)]]+\lambda F[\log \alpha-\mu(1)+1+\log n], \\
z_{\lambda}\left(X_{\lambda}\right)=\sum_{i=1}^{n-1} \lambda \mu(i)\left[\sum_{e \in \Omega^{+}(i)} x_{\lambda}(e)-\sum_{e \in \Omega^{-}(i)} x_{\lambda}(e)\right] \\
+\lambda \mu(1)\left[\sum_{e \in \Omega^{+}(1)} x_{\lambda}(e)-\sum_{e \in \Omega^{-}(1)} x_{\lambda}(e)-F\right] \\
+\lambda \mu(n)\left[\sum_{e \in \Omega^{+}(n)} x_{\lambda}(e)-\sum_{e \in \Omega^{-}(n)} x_{\lambda}(e)\right]+\lambda F(1+\log n), \\
z_{\lambda}\left(X_{\lambda}\right)=-F \lambda \log \omega(n)+\lambda F[1+\log n] .
\end{gathered}
$$

R.A.I.R.O. Recherche opérationnelle/Operations Research 


\section{Cas général}

Supposons que seule la condition $(A)$ soit vérifiée.

THÉORÈME $6:(a)$ L'inverse $B(\lambda)=\left\|b_{i j}(\lambda)\right\| \in R^{n \times n}$ de la matrice $I-A(\lambda)$ existe et $B(\lambda) \geqq 0, b_{i j}(\lambda)>0 \Leftrightarrow \exists$ un chemin d'origine $v_{i}$ et d'extrémité $v_{j}$.

(b) Supposons qu'il existe un chemin d'origine $v_{i}$ et d'extrémité $v_{j}$. Soit $A_{\lambda}$ l'affectation exponentielle pour un trafic d'intensité $F$ entre ces deux sommets. $A_{\lambda}$ produit sur chaque arc e d'origine $v_{l}$ d'extrémité $v_{k}$ un trafic:

$$
x_{\lambda}(e)=\frac{\bar{c}_{\lambda}(e) b_{i l}(\lambda) b_{k j}(\lambda)}{b_{i j}(\lambda)} . F .
$$

(c) La valeur du programme convexe associé à l'affectation exponentielle entre $v_{i}$ et $v_{j}$ est alors :

$$
z_{\lambda}=-F \cdot \lambda \log b_{i j}(\lambda)+\lambda F[1+\log n] .
$$

Démonstration: Voir [7].

\section{Remarques}

(a) Par le précédent théorème, la valeur $z_{\lambda}$ du programme $L_{\lambda}$ s'obtient par la résolution d'un seul système linéaire : $\left[I-A^{T}(\lambda)\right] \omega=u_{1}$. Pour obtenir la solution $X_{\lambda}$ il faut en plus résoudre le système $[I-A(\lambda)] \omega^{\prime}=u_{n}$. D'autre part, la matrice $B(\lambda)$ fournit directement la solution de tous les programmes $L_{\lambda}$ pour tous les couples $\left(v_{i}, v_{j}\right)$ origine-destination par les formules du théorème 6 .

(b) Si $\bar{c}_{i j}$ désigne la longueur cu plus court chemin entre $v_{i}$ et $v_{j}, \bar{c}_{i j}=\lim _{\lambda \rightarrow 0}-\lambda$ $\log b_{i j}(\lambda)$ par les théorèmes 4 et 6 .

$B(\lambda)$ représente donc une approximation de la matrice $\bar{C}=\left\|\bar{c}_{i j}\right\|$ des plus courts chemins.

(c) Soit $\beta$ le minimum des longueurs de tous les circuits du graphe $G$ avec les coûts initiaux. Par la condition $(A), \beta \geqq 0$.

Si $\beta>0$, définissons un nouveau système de coûts :

$$
c_{\lambda}(e)=c(e)-\lambda(1+\log n), \quad \forall e \in E .
$$

$\exists \lambda_{0}$ tel que pour $\lambda \leqq \lambda_{0}$ il n'y ait pas dans $G$ de circuits de longueur négative avec le système de coûts $c_{\lambda}(e)$. Les résultats précédents sont donc applicables avec les coûts $c_{\lambda}(e)$ pour $\lambda \leqq \lambda_{0}$. Notons alors que la quantité :

$$
\bar{c}_{\lambda}(e)=e^{-\left(c_{\lambda}(e) / \lambda\right)-(1+\log n)}
$$

vol. $15, \mathrm{n}^{\circ} 2$, mai 1981 
est égale :

$$
\bar{c}_{\lambda}(e)=e^{-\left(c_{\lambda}(e)+\lambda(1+\log n)\right) / \lambda}=e^{-c(e) / \lambda} .
$$

En particulier quand le graphe est sans circuit, on retrouve les quantités introduites au paragraphe II dans le cas de l'affectation de Dial.

Soit :

$$
f_{\lambda}^{\prime}(X)=f_{\lambda}(X)-\lambda(1+\log n)\left[\sum_{i=1}^{n} y(i)\right]
$$

et

$$
z_{\lambda}^{\prime}(X)=f_{\lambda}^{\prime}(X)+\sum_{e \in E} c(e) x(e)
$$

Notons que :

$$
z_{\lambda}^{\prime}(X)=f_{\lambda}(X)+\sum_{e \in E} c_{\lambda}(e) x(e)-\lambda(1+\log n) F
$$

Pour $\lambda<\lambda_{0}$ on peut donc remplacer la fonction $z_{\lambda}(X)$ par $z_{\lambda}^{\prime}(X)$ dans $L_{\lambda}$, les résultats précédent sont valables mais en remplaçant $c(e)$ par $c_{\lambda}(e)$ et en. posant :

$$
\bar{c}_{\lambda}(e)=e^{-c(e) / \lambda}, \quad \forall e \in E .
$$

La valeur du programme $L_{\lambda}$ étant alors $-\lambda F \log \omega(n)$.

6. Interprétation de l'affectation exponentielle $A_{\lambda}$ entre $\left\{v_{1}\right\}$ et $\left\{v_{n}\right\}$

LEMME 7 :

$$
B(\lambda)=\sum_{i=0}^{\infty}[A(\lambda)]^{i} \quad \text { avec } \quad[A(\lambda)]^{0}=I .
$$

Démonstration :

$$
\forall m, \quad B(\lambda)=\sum_{i=0}^{m}[A(\lambda)]^{i}+A^{m+1}(\lambda) B(\lambda),
$$

comme $A(\lambda) \geqq 0$ et $B(\lambda) \geqq 0$,

$$
A^{m+1}(\lambda) \times B(\lambda) \geqq 0 \Rightarrow \sum_{i=0}^{m}[A(\lambda)]^{i} \leqq B(\lambda) .
$$


Donc la série $[A(\lambda)]^{i}$ est convergente et $[A(\lambda)]^{m} \rightarrow 0$ quand $m \rightarrow+\infty$. $\Rightarrow[A(\lambda)]^{m+1} \times B \rightarrow 0$ quand $m \rightarrow+\infty . B(\lambda)$ est la limite de la série $[A(\lambda)]^{i}$.

Sur chaque arc considérons un coût $c_{\lambda}(e)=c(e)+\lambda[1+\log n]$.

Soit $T(i, j)$ l'ensemble des chemins d'origine $v_{i}$ d'extrémité $v_{j}$.

On vérifie alors que $b_{i j}(\lambda)=\sum_{t \in T(i, j)} e^{-c(t) / \lambda}$ où $c(t)$ est la longueur du chemin $t$ avec les coûts $c_{\lambda}(e)$.

THÉORÈME 7 : L'affectation exponentielle $A_{\lambda}$ revient à charger chaque chemin $t \in T(1, n)$ avec un trafic égal à $r(t)=\mu e^{-c(t) / \lambda}$.

Démonstration : $\mu$ est déterminé de telle façon que :

$$
\sum_{t \in T(1, n)} r(t)=F
$$

On en déduit :

$$
\mu=\frac{F}{b_{1 n}(\lambda)} .
$$

D'autre part, l'affectation décrite dans le théorème charge chaque arc $e$ d'origine $i$, d'extrémité $j$ d'un trafic :

$$
x_{\lambda}(e)=\sum_{t \in T(1, n ; e)} e^{-c(t) / \lambda}=\mu\left(\sum_{t \in T[1, o r(e)]} e^{-c(t) / \lambda}\right) \cdot e^{-c_{\lambda}(e) / \lambda} \cdot\left(\sum_{t \in T[e x(e) ; n]} e^{-c(t) / \lambda}\right) .
$$

Donc :

$$
x_{\lambda}(e)=F \times \frac{b_{1 i}(\lambda) \times b_{j n}(\lambda)}{b_{1 n}(\lambda)} \cdot c_{\lambda}(e)
$$

ce qui est bien le résultat de l'affectation exponentielle définie au théorème 6 .

\section{On a un théorème analogue au théorème 3 du paragraphe 1}

THÉORÈme : Soit $X=[x(e)]$ un vecteur trafic d'intensité $F$ entre $\left\{v_{1}\right\}$ et $\left\{v_{n}\right\}$. On supposera [en enlevant éventuellement les arcs pour lesquels $x(e)=0$ ] que $X>0$.

Posons :

$$
\bar{c}_{\lambda}(e)=\frac{x(e)}{y[e x(e)]}
$$

et :

$$
c(e)=-\lambda \log \bar{c}_{\lambda}(e)-\lambda(1+\log n) .
$$

vol. $15, \mathrm{n}^{\circ} 2$, mai 1981 
Avec les coûts $c(e)$ l'affectation exponentielle produit sur chaque arc un trafic égal à $x(e)$.

La démonstration est identique à celle du théorème 2 paragraphe II, en utilisant les formules données par le théorème 6 ( $\S \mathrm{III})$.

IV. MÉTHODES D'INVERSION DE MATRICE ET ALGORITHMES DE PLUS COURT CHEMIN

On a vu au paragraphe II, 4, remarque $(b)$ que $\bar{c}_{i j}=\lim _{\lambda \rightarrow 0}-\lambda \log b_{i j}(\lambda)$.

Les algorithmes de plus courts chemins sont donc liés aux algorithmes d'inversion de matrice dans la mesure où la matrice $\bar{C}=\left\|\bar{c}_{i j}\right\|$ des plus courts chemins apparaît comme la limite de $\left.\|-\lambda \log b_{i j} \lambda\right) \|$ quand $\lambda \rightarrow 0$.

Nous donnerons dans ce paragraphe les deux propriétés qui permettent de préciser ces liaisons.

DÉfinition : Soit une fonction continue $\lambda \rightarrow a(\lambda)$ vérifiant la propriété (P) : $\exists \lambda_{0}>0$ telle que $\forall \lambda<\lambda_{0}, a(\lambda) \geqq 0$.

Posons alors $r(a)=\lim _{\lambda \rightarrow 0, \lambda<\lambda_{0}}[-\lambda \log a(\lambda)]$. [On posera $\log (0)=-\infty$.]

Notons qu'au voisinage de $0, a(\lambda)$ peut s'écrire :

$$
a(\lambda)=e^{-(r(a) / \lambda)+s+\varepsilon(\lambda)} \quad \text { avec } \quad \varepsilon(\lambda) \rightarrow 0 \text { quand } \lambda \rightarrow 0 .
$$

Deux fonctions $a(\lambda)$ et $b(\lambda)$ vérifiant la propriété $(\mathrm{P})$ sont équivalentes et on notera $a(\lambda) \sim b(\lambda)$ si $r(a)=r(b)$.

Cette définition s'étend à une matrice $B(\lambda)=\left\|b_{i j}(\lambda)\right\|$ où $b_{i j}(\lambda)$ vérifient la propriété $(\mathrm{P}): r(B)=\left\|r\left(b_{i j}\right)\right\|$.

On a le résultat suivant :

LEMME 1 : Soient $a_{1}(\lambda), a_{2}(\lambda)$ fonctions satisfaisant la propriété $(\mathrm{P})$.

Si $a_{3}(\lambda)=a_{1}(\lambda)+a_{2}(\lambda)$,

$$
r\left(a_{3}\right)=\min \left[r\left(a_{1}\right), r\left(a_{2}\right)\right] .
$$

Si $a_{3}(\lambda)=a_{1}(\lambda) a_{2}(\lambda)$,

$$
r\left(a_{3}\right)=r\left(a_{1}\right)+r\left(a_{2}\right)
$$

Évident. 
Lemme 2 : Soient $B_{1}(\lambda)=\left\|b_{i j}^{1}(\lambda)\right\|$ et $B_{2}(\lambda)=\left\|b_{i j}^{2}(\lambda)\right\|$ des matrices satisfaisant la propriété $(\mathrm{P})$.

$$
\begin{gathered}
r\left(B_{1}+B_{2}\right)=\min \left[r\left(B_{1}\right), r\left(B_{2}\right)\right], \\
r\left(B_{1} \cdot B_{2}\right)=r\left(B_{1}\right)+r\left(B_{2}\right) .
\end{gathered}
$$

En définissant les opérations $\oplus$ et $\otimes$ par $: r_{1} \oplus r_{2}=\min \left(r_{1}, r_{2}\right)$ et $r_{1} \otimes r_{2}=r_{1}+r_{2}$ on retrouve la structure algébrique introduite et étudiée par plusieurs auteurs $[1,8]$, la fonction $r(a)$ permettant d'associer les opérations + et $\oplus$ d'une part et $\times$ et $\otimes$ d'autre part.

Dans [8] une étude précise de ces relations entre algorithmes de plus court chemin avec les opérations $\oplus, \otimes$ et les méthodes d'inversion de matière avec les opérateurs,$+ \times$ est faite montrant que tous les algorithmes classiques de plus court chemin s'interprètent à la limite comme certains algorithmes d'inversion de matrice (soit exact, soit itératif).

REMARQUe : Il faut noter cependant que toutes les autres méthodes de résolution de système linéaire ou d'inversion de matrices s'appliquent au problème de l'affectation exponentielle sans pouvoir nécessairement s'interpréter à la limite, comme un algorithme de plus court chemin. Citons par exemple des méthodes itératives de projections orthogonales successives sur les hyperplans constitués par chaque contrainte ([5], chap. III, $\S \mathrm{V}, \mathrm{p}: 120)$ ou les méthodes de calcul de minimum d'une forme quadratique ([5], chap. III, § IV, p. 112).

\section{v. CONCLUSION}

Les méthodes exactes d'inversion de matrices nécessitent un nombre d'itérations de l'ordre de $O\left(n^{3}\right)$ ou $n=|V|$ comme les algorithmes de calcul de tous les plus courts chemins. Cependant les méthodes d'inversion de matrice sont plus compliquées que les algorithmes de plus court chemin comme le montrent à l'évidence les comparaisons faites au paragraphe IV. $[$ Les méthodes sont aussi longues à condition de calculer la matrice $B(\lambda)=I+\sum_{i=1}^{n} A^{i}(\lambda)$.

La matrice $B(\lambda)$ a cependant de nombreux avantages par rapport à la matrice $\bar{C}$ des plus courts chemins.

$1^{\circ}$ On peut connaître directement à partir de $B(\lambda)$ toutes les affectations exponentielles entre tous les sommets par les formules du théorème 5 , paragraphe II. 
$2^{\circ}$ Notons surtout que la matrice $B(\lambda)$ contient beaucoup plus d'informations que la matrice $\bar{C}$. Ceci se remarque sur l'interprétation faite au paragraphe III, 6 .

En particulier, on peut reconstruire la matrice $A(\lambda)$ à partir de $B(\lambda)$ alors qu'il n'est pas toujours possible de reconstituer la matrice $C$ à partir de $\bar{C}$ comme le montre l'exemple suivant: $G$ est un graphe à trois sommets $v_{1}, v_{2}, v_{3}$ et trois arcs $e_{1}=\left(v_{1}, v_{2}\right) ; e_{2}=\left(v_{2}, v_{3}\right) ; e_{3}=\left(v_{1}, v_{3}\right)$.

Les systèmes de coûts $\left[c\left(e_{1}\right)=1, c\left(e_{2}\right)=1, c\left(e_{3}\right)=2\right]$ et $c^{\prime}\left(e_{1}\right)=1, c^{\prime}\left(e_{2}\right)=1$, $c^{\prime}\left(e_{3}\right)=5$ fournissent la même matrice de plus courts chemins :

$$
\bar{c}_{12}=1, \quad \bar{c}_{23}=1, \quad \bar{c}_{13}=2, \quad \bar{c}_{21}=\bar{c}_{32}=\bar{c}_{31}=+\infty .
$$

Grâce à ces dernières remarques, les méthodes de calcul développées dans cet article peuvent trouver d'intéressantes applications à divers problèmes liés à des calculs de plus court chemin. Une prochaine publication étudiera quelques-unes de ces extensions.

\section{BIBLIOGRAPHIE}

1. B. A. CARre, An Algebra for Network Routing Problems, J. Inst. Math. Appl., vol. 7, 1971, p. 273-294.

2. G. B. Dantzig, Linear Programming and Extensions, Princeton University Press, 1963.

3. G. B. Danrzig, On a Shortest Route Through a Network, Manag. Sc., vol. 6, 1960, p. $187-189$.

4. R. B. Dial, A Probabilistic Multipath Traffic Assignment Model which Obviates Path Enumeration, Transp. Res., vol. 5, 1971, p. 83-111.

5. E. Durand, Solutions numériques des équations algébriques, tome II, Masson, Paris, 1961.

6. P. H. FARGIER, Une méthode de détermination de réseau optimal, février 1977, I.R.T.

7. J. FONLUPT, L'affectation exponentielle et le probleme du plus court chemin dans un graphe, Séminaire Analyse numérique $n^{\circ} 275$, Mathématiques appliquées, Université scientifique et médicale de Grenoble.

8. M. GondRAN, Algèbre linéaire et cheminement dans un graphe, R.A.I.R.O., 1975, p. 77-98.

9. R. T. Rockafellar, Convex Analysis, Princeton University Press, 1970. 